\title{
SCHEIN ODER NICHT-SCHEIN? ZUR INSZENIERUNG VON AUTHENTIZITÄT AUF DER BÜHNE
}

\section{Ralf von Appen}

Ziel einer wissenschaftlichen Auseinandersetzung mit dem Wert der Authentizität kann es nicht sein zu entscheiden, wer oder was wahrhaft authentisch ist. Einen verbindlichen, konsensuell akzeptierten Maßstab dafür wird es nicht geben, da mit dem Authentizitäts-Begriff verschiedene, einander mitunter widersprechende Werte angesprochen werden, die zudem noch historischem Wandel unterworfen und so nicht auf einen stabilen gemeinsamen Nenner zu bringen sind. Diese konkurrierenden Wertvorstellungen ergeben sich aus den unterschiedlichen persönlichen und sozialen Bedürfnissen der Hörerinnen und Hörer und haben somit allesamt ihre Berechtigung. Das Bemühen sollte sich vielmehr darauf richten, verschiedene Konstruktionen und Zuschreibungen von Authentizität zu dokumentieren und darin wiederkehrende Muster zu erkennen, um die dahinter verborgenen Ideale, Bedürfnisse, Identifikations- bzw. Distinktionsbemühungen etc. offen zu legen. Damit verbunden ist das kulturwissenschaftliche Interesse, gesellschaftliche und individuelle Funktionen von Musik zu verstehen. Ein weiteres Ziel ist es daneben, durch die Schärfung des Bewusstseins für den Inszenierungscharakter medial vermittelter Authentizitätskonstruktionen zum aufgeklärten Umgang mit ökonomisch und ideologisch motivierten Inszenierungen beizutragen.

Mit diesen Absichten werde ich zunächst die zentralen Begriffe Authentizität, Liveness und Inszenierung diskutieren, um dann anhand aktueller Beispiele verbreitete Authentifizierungsstrategien speziell bei Live-Auftritten untersuchen. Auf der Bühne nämlich zeigt sich der Konflikt dieser drei Ideen am deutlichsten. 


\title{
I. Authentizität - Liveness - Inszenierung
}

\begin{abstract}
Authentizität
Authentizität ist ein ethisches Ideal, das auf den Werten der Ehrlichkeit, der Treue und der Konsequenz basiert, sowohl sich selbst wie auch anderen gegenüber. Bezogen auf zwischenmenschliche Beziehungen bringt es das essentielle Bedürfnis zum Ausdruck, nicht getäuscht und nicht enttäuscht zu werden. Als individual-ethischem Anspruch liegen ihm die Bedürfnisse nach Selbstverwirklichung und individueller Freiheit zugrunde und damit die Wünsche, das eigene Potential ausschöpfen und sich den persönlichen Idealen entsprechend verhalten zu können.

Im Kontext der Musik sind zumindest folgende vier Dimensionen von Bedeutung:
\end{abstract}

\section{a) Persönliche Authentizität}

Als authentisch gilt, wem es gelingt, innere Überzeugungen und äußeres Handeln in Übereinstimmung zu bringen. Menschen, die so ihrem inneren Kompass folgen, hält man für ehrlich und man weiß, was man von ihnen zu erwarten hat. Dieser Anspruch richtet sich seit Mitte der 1960er Jahre auch an Rock- und Popmusiker. Zu dieser Zeit begann man sie weniger als Entertainer denn als Künstler anzusehen, wobei das Künstlerbild - ursprünglich vermittelt durch britische Art Schools und das Vorbild der Beat Poets sowie von Dichtern wie Baudelaire, Rimbaud, Keats oder Blake - starke romantische Züge trägt (vgl. Frith/Horne 1987, Pattison 1987, Appen 2013). John Lennon, Jim Morrison oder Jimi Hendrix wurden von ihren Anhängern - im Gegensatz zu den Stars der Elterngeneration - für unangepasste Individuen mit besonderer Persönlichkeit gehalten, die nur ihren eigenen Überzeugungen verpflichtet sind und sich unabhängig von gesellschaftlichen Autoritäten und marktwirtschaftlicher Einflussnahme selbst verwirklichen. Damit wurden sie im Kontext der politischen Auseinandersetzung der Baby-BoomerGeneration mit dem »Establishment « zu in die Zukunft weisenden Vorbildern für eine bessere Gesellschaft, in der das Ideal des von autoritärer Ordnung befreiten Menschen mit »befreitem Bewusstsein« verwirklicht sein würde und man ein Leben würde führen können, »das nicht in der Erfüllung von Rollenerwartungen, der Entsprechung an Verhaltenskonventionen oder der Umsetzung von traditionell bestimmten Lebensplänen aufgeht« (Menke 2005: 309). Mit dieser vermeintlichen Unabhängigkeit von gesellschaftlichen Zwängen wurde die Erwartung verbunden, dass die Musiker authentisch blei- 
ben, sich also radikal selbst verwirklichen und die idealisierte Freiheit ausleben, statt sich denselben Kompromissen zu unterwerfen, die das Publikum tagein, tagaus eingehen muss.

Besonders verbreitet sind solche Ideale seitdem in allen Spielarten der populären Musik, die sich als gegenkulturell oder rebellisch vom so genannten Mainstream abgrenzen wollen (zur Bedeutung von Authentizität für Punk-Fans S. z.B. Lewin/Williams 2009). Gerade weil jede Musik, die größere Verbreitung finden will, an marktwirtschaftliche Strukturen gebunden ist und so immer im Verdacht steht, sich diesen zu beugen, ist das Kriterium der Authentizität hier so wichtig. Als größtmögliche Enttäuschung gilt es daher, wenn Musiker ihre Musik oder ihr Image durch Aussicht auf Ruhm und kommerziellen Erfolg den Erwartungen der Mehrheit zu sehr anpassen (»selling out«, Beispiele in Appen 2007: 118ff.). Als besonders authentisch gilt im Gegenzug, wer mit (vermeintlich) unabhängigen Labels außerhalb großkapitalistischer Strukturen arbeitet und so viele Entscheidungen und Produktionsschritte wie möglich selbst verantwortet (das »Do-It-Yourself «Ideal).

Aber auch jenseits dieser freiheitlichen Rock-Ideologie zeigen Fans großes Interesse am Privatleben der Stars und an der Frage, inwiefern sich öffentlich vermitteltes Image und »tatsächliche« Persönlichkeit decken. Gerade, wenn man Stars zum Vorbild wählt und sich über sie identifiziert, will man wissen, woran man ist. Authentizität wird so auch Popstars mit großen Mainstream-Erfolgen zugesprochen, wenn sie trotz Ruhm und Reichtum den Eindruck erwecken, »natürlich « und »sich selbst treu « geblieben zu sein (s. den Abschnitt über Adele, S. 60ff).

\section{b) Sozio-kulturelle Authentizität}

Mit dem Ideal der persönlichen Authentizität verbunden, aber anders motiviert, ist der Anspruch, dass Musiker den Werten ihres Publikums, insbesondere der lokalen oder sozialen Subkultur, aus der sie hervorgegangen sind, treu bleiben. Sie sollen sich auch im Falle von Erfolg und gesellschaftlichem Aufstieg mit dieser Subkultur identifizieren, um nicht im Gegenzug diejenigen zu kompromittieren, die sich von ihnen repräsentiert fühlen. Auch dieses Ideal findet sich naturgemäß vor allem (aber keineswegs nur) dort, wo Bands und Fans sich vom »Mainstream« abgrenzen wollen (vgl. Appen 2007: 122ff.).

An dem Konflikt, in den Bob Dylan mit seinem Auftritt mit Band und E-Gitarre beim Newport Festival 1965 geriet, zeigt sich beispielhaft, dass persönliche Authentizität (= das Verfolgen der eigenen Werte) und sozio- 
kulturelle Authentizität (= die Übereinstimmung mit teilkulturellen Werten) sich mitunter nicht vereinen lassen: Den einen galt Dylan als »sich selbst treu« im Sinne der persönlichen Authentizität, weil er sich nicht von Fans, Förderern und Kritikern vereinnahmen ließ, sondern seiner persönlichen Überzeugung folgte und sich künstlerisch weiterentwickelte. Seine Gegner aus der Folk-Revival-Szene, die sich von Dylan bis dahin repräsentiert sahen und denen er seine Karriere zu verdanken hatte, sahen in der Elektrifizierung dagegen den ökonomisch motivierten Versuch, an die Erfolge der Beatles und der Byrds anzuknüpfen. In ihren Augen verriet Dylan mit dieser Anpassung an eine oberflächliche Mode die grundlegenden politischen und ethischen Werte der Folk-Bewegung: Distanz zur Unterhaltungsindustrie und zum Establishment, Ernsthaftigkeit, Traditionsbewusstsein und vor allem soziales Engagement (vgl. Nelson 1965).

\section{c) Handwerkliche Authentizität}

Entsprechend dem ursprünglichen Wortsinn des griechischen Verbs authéntein (= selbst ausführen, eigenständig tun; s. Stimpfle 2011: 161) bzw. dem Adjektiv authentikós (= von eigener Hand ausgeführt, ebd.) betrifft diese Dimension das Bedürfnis, nicht über die Urheberschaft und die handwerklichen Leistungen von Musikern getäuscht zu werden. Abgelehnt und sanktioniert werden dementsprechend Plagiate und - je nach Situation und Genre - Lip-Synching, der Einsatz von Playback oder Auto-Tune und andere Vortäuschungen von Kompetenz. Prominente Beispiele sind die Aberkennung des Grammy-Awards an Milli Vanilli 1990 (s. Friedman 1993) oder die vom Spiegel erhobenen Vorwürfe, die Rolling Stones würden bei ihren Konzerten auf Playbacks zurückgreifen (N.N. 1995). In diesem Sinne ist auch der Hinweis auf den frühen Queen-Alben, die Band habe bei den Aufnahmen keinerlei Synthesizer verwendet, als Absage an »maschinelle« Hilfe zu verstehen. Hinter der Empörung über die Vortäuschung nicht vorhandener Kompetenz steht zum einen der Arbeitsethos des »ehrlichen Handwerks«, der in der Country Music, im Rock und insbesondere im Heavy Metal verbreitet ist (Diaz-Bone 2002: 408 u. 410): Wertschätzung für eine Arbeit solle man sich durch hart errungene Qualität verdienen, nicht durch falsche Angaben erschleichen. Zum anderen spielt auch hier der Aspekt der Identifikation eine Rolle: Wie soll man sich von einem Musiker repräsentiert fühlen, der nicht hundertprozentig mit dem zu identifizieren ist, was er zu sein vorgibt, dessen Musik nicht einmal ihn selbst repräsentiert? Und nicht zuletzt gilt »von eigener Hand gemachte« Musik als Voraussetzung für emotionale Authentizität. 
d) Emotionale Authentizität

Ebenfalls romantischen Ursprungs ist der Anspruch, dass Musik, die emotional bewegt, ihren Ursprung im persönlichen Leben der Musiker haben soll. Dieser Wunsch nach einem authentischen emotionalen Ausdruck basiert auf drei Grundannahmen:

Erstens wird Musik (mehr oder weniger bewusst) als kulturelles Medium verstanden, das der Kommunikation wichtiger Lebens-Erfahrungen dient. Menschen nutzen Musik, um eigene emotionale Erfahrungen zu denen anderer in Beziehung zu setzen, um zu imaginieren, wie es sich anfühlt, in bestimmten Situationen zu sein, um mit ihren Gefühlen nicht allein zu sein und zu hören, wie es anderen in Freud oder Leid ergeht. Wenn die emotionale Dimension von Musik also wichtig für das eigene Leben wird, dann will man sich darauf verlassen können, dass die bevorzugte Musik tatsächlich etwas Echtes, Wahres über das Leben vermittelt und nicht bloßer Fiktion entspringt.

Dies ist vor allem dann möglich, so die zweite Grundannahme, wenn der emotionale Ausdruck autobiographisch verwurzelt ist. Anders als von Schauspielern glaubt und fordert man von Pop- und Rockmusikern kaum, dass sie sich für verschiedene Songs in verschiedene Rollen versetzen, sondern dass sich der emotionale Ausdruck ihrer Songs aus ihrem eigenen Gefühlsleben speist, sie also tatsächliche Facetten ihrer Persönlichkeit zum Ausdruck bringen (entsprechende Aussagen von Musikern und Fans s. Appen 2007: 129f.). Entsprechend wird in vielen Stilbereichen erwartet, dass Interpreten ihre Songs selbst schreiben oder gecoverten Stücken zumindest ihren persönlichen Stempel aufdrücken. Das medial vermittelte Wissen um Drogensucht, Ghetto-Hintergrund, unglückliche Liebe oder Erfahrungen mit dem Tod authentifiziert Musiker wie Kurt Cobain, Herbert Grönemeyer, 50 Cent oder Johnny Cash zusätzlich. Es beseitigt das Misstrauen, dass jemand über ein Leid klagt, das er nicht erfahren hat, und so nicht wirklich in der Lage ist, Einblicke in essentielle Lebenssituationen zu vermitteln, mit denen es sich auseinanderzusetzen lohnt.

Drittens herrscht bei einem nicht geringen Teil des Publikums weiterhin die romantische Vorstellung, dass Emotionen geeigneter seien, etwas Wahres über menschliche Verhältnisse zur Welt zu vermitteln als Sprache und Vernunft, »dass es eine sechte Wahrheit< nur im Fühlen und nicht im Denken gibt« (Funk 2011: 231). Gerade durch Gestik, Mimik und eben Musik zum Ausdruck gebrachte Emotionen gelten dabei nach wie vor als unmittelbarer, unverfälschter Ausdruck des Selbst: »Feelings and emotions are keys for unlocking who I am, my authenticity, how I perceive and how I discover my 
>real self « (McCarthy 2009: 241). Mit dieser Überzeugung geht eine Ablehnung von technischen Hilfsmitteln und zu großer rationaler Einflussnahme auf den Schaffensprozess einher: Das Emotionale solle nicht durch zuviel Perfektion, durch Auto-Tune oder andere Computertechnik zerstört werden, Musik solle lieber ungeschliffen und »natürlich « klingen als glatt, unmenschlich und ausdruckslos. ${ }^{1}$ Diese Werthaltung brachte Dave Grohl von den Foo Fighters in seiner Dankesrede für den 2012 gewonnenen Grammy in der Kategorie »Best Rock Album« auf den Punkt:

»This is a great honor. Because this record was a special record for our band. Rather than, rather than go to the best studio in the world down the street in Hollywood and rather than use all the fanciest computers that you can buy, we made this one in my garage with some microphones and a tape machine. [...] To me this award means a lot because it shows that the human element of making music is what's most important. Singing into a microphone and learning to play an instrument and learning to do your craft, that's the most important thing for people to do. It's not about being perfect, it's not about sounding absolutely correct, it's not about what goes on in a computer. It's about what goes on in here [zeigt auf die Brust] and what goes on in here [zeigt auf seinen Kopf] « (Grohl 2012). ${ }^{2}$

Weitere Dimensionen der Authentizität sind denkbar, etwa die »cultural« (Barker/Taylor 2007: X) oder »third person authenticity « (Moore 2002: 214218), die einen »echten« Einblick in eine bestimmte historische oder ethnische Kultur-Tradition verspricht. Sie spielen in der Live-Situation aber eine geringere Rolle, weshalb sie hier zu vernachlässigen sind. ${ }^{3}$

1 Die Einsicht, dass Intimität und Nähe erst durch die Erfindung des Mikrophons auf Tonträgern vermittelt werden konnten (s. Dibben 2009: 319-321) oder dass meist viel technischer Aufwand nötig ist, um den Eindruck des »Natürlichen « zu erzeugen, hat sich jenseits professioneller Kreise noch nicht durchsetzen können.

2 Entsprechend veröffentlichten die Foo Fighters zuvor ein Online-Video, in dem sie das gesamte Album Wasting Light in ihrem Studio ohne Publikum live spielen, um ihre handwerkliche Authentizität unter Beweis zu stellen (s. Foo Fighters 2011).

3 Andere Systematisierungen verschiedener Authentizitäts-Dimensionen finden sich bei Fornäs (1994: 168: "social«, "subjective« und »cultural or metaauthenticity«), Moore (2002: »authenticity of expression«, »of execution«, »of experience«) sowie Barker/Taylor (2007: X: »personal«, »representational« und »cultural authenticity«). Trotz teilweise identischer Bezeichnungen unterscheiden sich die dortigen Bestimmungen inhaltlich von den hier vorgeschlagenen Dimensionen. 


\section{Liveness}

Eine Aufführung, so definiert Erika Fischer-Lichte (2005a: 16), ist ein »Ereignis [...], das aus der Konfrontation und Interaktion zweier Gruppen von Personen hervorgeht, die sich an einem Ort zur selben Zeit versammeln, um in leiblicher Ko-Präsenz gemeinsam eine Situation zu durchleben. "Wenn auch die primären Handlungen bei einer Aufführung von den Akteuren auf der Bühne ausgehen, so haben die Zuschauer in dieser Ko-Präsenz doch eine konstitutive Rolle, da ihre Reaktionen (Zwischenrufe, Mitsingen und -klatschen, Tanzen, Raunen, Feuerzeuge hochhalten, mit dem Handy fotografieren, Lachen, den Saal verlassen...) - bzw. deren unerwartetes Ausbleiben - das Erleben und Handeln der anderen Zuschauer und der Akteure unmittelbar beeinflussen. »In diesem Sinne läßt sich behaupten «, so Fischer-Lichte (2004: 59), »daß die Aufführung von einer selbstbezüglichen und sich permanent verändernden feedback-Schleife hervorgebracht und gesteuert wird.«

Die besonderen Erfahrungsqualitäten von Aufführungen soll der Begriff Liveness zusammenfassen. ${ }^{4}$ Vor allem, dass solche Ereignisse flüchtig und wesentlich durch die Interaktion von Publikum und Aufführenden geprägt sind, dass sie jederzeit durch Fehler oder Unvorhergesehenes beeinflusst werden können und somit nicht vollständig planbar und nicht exakt wiederholbar sind, sorgt für Spannung und Überraschungen - und damit für ein intensiviertes Erleben der eigenen Gegenwart (vgl. Kolesch 2005: 188f.). New York Times-Kritiker Jon Pareles (1990) bringt diesen Reiz eines LiveKonzerts auf den Punkt: »If I wanted flawlessness, I'd stay home with the album. The spontaneity, uncertainty and ensemble coordination [...] are exactly what I go to concerts to see; the risk brings the suspense, and the sense of triumph, to live pop."

Bezogen auf die verschiedenen Dimensionen der Authentizität bietet die Live-Aufführung die seltene Möglichkeit, den Musikern einigermaßen unmit-

4 Philip Auslanders (1999: 32-35, 50 u. 85-94) zur Hoch-Zeit des Musikfernsehens geäußerte These, Erfahrungen von authentischer Liveness seien in unserer stark »mediatized culture nicht mehr möglich, da ihre Wahrnehmung unweigerlich durch unsere Erfahrungen mit technischen Medien kontaminiert sei, überzeugt mich in ihrem Absolutheitsanspruch vor dem Hintergrund eigener Konzerterfahrungen nicht. Selbst wenn dies so wäre, änderte es nichts an der von FischerLichte beschriebenen prinzipiellen Interaktivität von Aufführungen - ein ästhetischer Reiz, den Auslander offenbar übersieht (vgl. ebd.: 54-58). Zur Kritik an Auslander siehe auch Pattie (2007: 21-39). 
telbar persönlich zu begegnen und deren Authentizitätsversprechen so auf besondere Art überprüfen zu können:

- Die sozio-kulturelle Authentizität der Musiker, das Teilen gemeinsamer Werte, kann sich in der Nähe der Stars zu den Fans, in der Kommunikation mit dem Publikum, in der Kleidung der Musiker, den Ticketpreisen und in der Songauswahl zeigen.

- Die Konzertlänge, Ansagen und das Verhalten der Musiker auf der Bühne erlauben Rückschlüsse auf ihre persönliche Authentizität, etwa wenn der Eindruck von Spielfreude und Spaß an der Interaktion mit dem Publikum entsteht.

- Die handwerkliche Authentizität ist besser zu beurteilen, wenn das Spiel der Musiker direkt beobachtet werden kann und Netz und doppelter Boden der Studioproduktion wegfallen (bezogen auf Heavy Metal s. DiazBone 2002: 409).

- Und in der (relativ) unmittelbaren Begegnung mit den Musikern glaubt man, die emotionale Authentizität besser als in durchgestylten Studioproduktionen beurteilen zu können, bspw. an der Stimme, der Mimik und Gestik.

Vor allem die beiden letzten Dimensionen hat Lawrence Grossberg im Blick, wenn er die Bedeutung von Live-Konzerten für die Authentifizierung von Musikern hervorhebt:

»Die Wichtigkeit von Live-Aufführungen liegt eben in dem Umstand, dass man nur hier die eigentliche Produktion des Sounds sehen kann, und die in der Stimme verpackte emotionale Arbeit. Die Forderung nach Live-Aufführung drückte immer das Verlangen nach sichtbarer Markierung (und Beweis) der Authentizität aus« (Grossberg 2010: 202).

\section{Inszenierung}

Von der Aufführung grenzt Fischer-Lichte die Inszenierung als konzeptuelle Planung ab, die jeder Aufführung zwingend vorausgehen muss:

»Inszenierung lässt sich [...] als der Prozess beschreiben, in dem allmählich die Strategien entwickelt und erprobt werden, nach denen was, wann, wielange, wo und wie vor den Zuschauern in Erscheinung treten soll. Inszenierung lässt sich entsprechend [...] als Erzeugungsstrategie bestimmen. [...] Daraus folgt auch, dass zwischen Inszenierung und Aufführung unbedingt zu unterscheiden ist. Es sind erst die Wahrnehmung der Zuschauer und deren Reaktion auf das Wahrgenommene, welche die Aufführung entstehen lassen. Das, was im Prozess der Inszenierung geplant und festgelegt ist, wird 
sich daher keineswegs allabendlich genau gleich wiederholen« (FischerLichte 2005b: 148). ${ }^{5}$

Dass eine Inszenierung zwingend »überall da gegeben [ist], wo eine Aufführung stattfinden soll« (ebd.), weil Aufführungen mit mehreren Akteuren sorgfältig vorbereitet und einstudiert werden müssen, bedeutet allerdings nicht, dass alle Aufführungen im selben Maße durch Planung vorherbestimmt sind. Es kann gerade das Ziel von Inszenierungen sein, Momente der Unbestimmtheit entstehen zu lassen, damit sich »Nicht-Geplantes, NichtInszeniertes, Nicht-Vorhersagbares in der Aufführung ereignen kann, auch wenn manche [...] Inszenierung versuchen mag, diese Frei- und Spielräume so weit wie möglich einzuschränken« (Fischer-Lichte 2004: 327).

Bei der Übertragung dieser Bestimmung von der Theaterbühne, über die Fischer-Lichte spricht, auf Musikaufführungen ist zusätzlich zu bedenken, dass sich die notwendigen Proben und Erzeugungsstrategien nicht zwingend auf den visuellen Bereich erstrecken müssen, worauf Martin Seel hingewiesen hat. Daher sollte im Falle der Aufführung von Musik erst dann von Inszenierung gesprochen werden, »wenn ihre Aufführung mit einer für ihren Charakter als Musik relevanten szenischen Bewegung verbunden ist - wie es in er populären Musik fast immer, in der Avantgarde-Musik nicht selten der Fall ist « (Seel 2007: 79), wenn es also ein Konzept für die visuelle Präsentation gibt. Zu sehen und als bedeutungstragend $z u$ interpretieren sind dann neben dem Bühnendesign Bewegungen, Posen und Gesten von Musikern, Beleuchtung, Kleidung oder gar Kostüme, Videoeinspielungen, Requisiten oder Choreographien. Um nun aber zu entscheiden, wann es sich bei dieser visuellen Ebene um eine inszenierte, also um eine auf Erzeugungsstrategien basierende handelt, muss man berücksichtigen, dass - wie letztlich auch auf der Theaterbühne - nicht alle sichtbaren Elemente zwangsläufig Teil einer bewussten Strategie sein müssen, was Seel in seiner Definition von Inszenierung herausstellt: »Inszenierungen sind ein künstliches, ein artifizielles Verhalten und Geschehen, das sich als ein solches, von bloß kontingenten, bloß konventionellen oder bloß funktionalen Vollzügen unterscheidet (ebd.: 71).

Bezogen auf Konzerte können bspw. die Aufstellung und der Bewegungsradius der Musiker auf der Bühne weniger Teil einer visuellen Inszenierung als vorrangig der notwendigen Funktionalität geschuldet sein, dass sie sich untereinander sehen, hören und verständigen müssen. Entsprechend sind

5 Da er diese wichtige Differenzierung von Aufführung und Inszenierung verwischt, verzichte ich auf den durch inflationären Gebrauch unscharf gewordenen Begriff der Performance, zumal sich "staging « als englischer Begriff für Inszenierung nicht im selben Maß durchgesetzt hat. 
Instrumente, Bekleidung, Verstärker, Monitorboxen etc. nur zum Teil als strategisch in Szene gesetzte Requisiten anzusehen. Durch Konvention begründet können Bühnenhandlungen und -gestaltungen sein, die so gewöhnlich sind, dass sie kaum als bewusste Inszenierungen intendiert oder gedeutet werden bzw. erst dann als solche auffällig werden, wenn bewusst auf sie verzichtet wird: etwa das Verbeugen und das Bedanken für Applaus, die Abtrennung eines Zugaben-Teils von der Hauptshow, typische Gesten der Publikumsanimation, die Illumination von Sängern oder Solisten. Und ob eine Aufführung durch Zufall beeinflusst war, kann das Publikum erst entscheiden, wenn es mehrere Aufführungen gesehen hat (was sich in Zeiten von YouTube relativiert).

Diese Vielfalt der Deutungsmöglichkeiten hat zur Folge, dass - wie im alltäglichen Leben - nie ganz sicher entschieden werden kann, ob und in welchem Grade man es mit einem inszenierten Geschehen zu tun hat (vgl. ebd.: 81). Das bedeutet im Gegenzug, dass auch die Akteure auf der Bühne nicht sicher sein können, ob und inwiefern das Aufgeführte als Inszenierung wahrgenommen wird oder nicht. Dies hat zwei sehr wichtige Folgen: Erstens haben die Akteure die Möglichkeit, so zu inszenieren, dass die Erzeugungsstrategie dem Publikum nicht auffällt, dass also alle Bühnenhandlungen als spontan und natürlich wahrgenommen werden. Gerade bezüglich der Konstruktion von Authentizität sind die wirkungsvollsten Inszenierungen sicher die, die nicht als solche auffällig werden. Und zweitens: Selbst wenn die Musiker sich bemühen, nichts zu inszenieren und sich auf der Bühne so spontan und natürlich wie nur möglich zu verhalten, garantiert dies nicht, dass das Publikum das Geschehen nicht doch als Inszenierung interpretiert. Selbst die treffendste Definition von Inszenierung hilft somit nicht bei der Entscheidung, ob und wie hochgradig im konkreten Fall inszeniert wurde. Das bleibt Interpretationssache.

Wenn im Popkonzert nun die Ideale der Authentizität und der Liveness mit Strategien der Inszenierung zusammentreffen, dann entsteht zwangsläufig ein Konflikt: Die Forderung nach Authentizität impliziert die Liveness-Ideale Spontaneität, Einmaligkeit und Unmittelbarkeit. Inszenierung bedeutet dagegen in aller Regel Planung statt Spontaneität, Wiederholbarkeit statt Einmaligkeit und anstelle der Unmittelbarkeit eine konzeptionelle Strategie, die zwischen den Akteuren und den Zuschauern steht. Folglich wird Inszenierung häufig als etwas Negatives angesehen. Sie wird kritisch bewertet als Manipulation des Publikums, als Trickserei, auch als visuelle Ablenkung von der Musik oder, wie Fischer-Lichte (2005b: 151) zu Argumentationszwecken 
überspitzt formuliert, als Herstellung von bloßem Schein, »von Täuschung, Lug und Trug «.

Wenn es im Konzert den Idealen der personalen, sozio-kulturellen und emotionalen Authentizität zufolge darum geht, sich mit den Personen auf der Bühne zu identifizieren, sich von ihnen repräsentiert zu fühlen und von ihnen etwas Wahres über das Leben zu erfahren, dann möchte man nicht Schauspielern begegnen, sondern »echten Menschen«, denn mit Schauspielern identifiziert man sich nicht im selben Maß. Insofern müssten Musiker es nach allen Möglichkeiten vermeiden, irgendetwas auf der Bühne zu inszenieren - was wie erwähnt nicht davor schützt, dass auch dies als Statement, als Strategie, gedeutet werden kann. Auch Understatement ist schließlich ein Statement.

Auf der anderen Seite bietet das Medium der Bühne nicht nur die Gelegenheit »Schein« zu präsentieren, sondern auch »Sein«. Inszenieren muss nicht »sich verstellen« bedeuten, es bietet auch die Möglichkeit, ein tatsächliches, reales Sein ungeschminkt, leicht gefiltert oder auch plakativ herauszustellen, also »Authentizität zur Erscheinung zu bringen« (FischerLichte 2000: 40). Inszenierung kann helfen - oder ist sogar notwendig -, um tatsächlich vorhandene Qualitäten zu demonstrieren, auf der Bühne also einen Rahmen zu schaffen, um sich vor aller Welt von seiner besten Seite, im besten Licht zu zeigen - und die andere Seite im Bühnendunkel zu verbergen.

Im folgenden sollen nun einige Beispiele zeigen, wie Musiker auf der Bühne mit diesem Konflikt, aber auch mit dieser Herausforderung umgehen: Welche Inszenierungs-Strategien lassen sich erkennen, welche Marker werden eingesetzt, um Authentizität - je nach Interpretation - vorzutäuschen oder zum Ausdruck zu bringen?

\section{Inszenierungen von Authentizität}

Die am weitesten verbreitetste Strategie ist, wie angedeutet, so zu inszenieren, dass Authentizitätseffekte entstehen, ohne dass der Inszenierungscharakter auffällig wird, das Geschehen auf der Bühne also möglichst natürlich und spontan wirkt. Ein hervorragendes Beispiel liefert die Inszenierung der Metallica-Welttournee der Jahre 1996/7, dokumentiert im Konzertfilm Cunning Stunts, für den Regisseur Wayne Isham die Aufnahmen zweier Auftritte in Fort Worth, Texas, montiert hat.

Gewöhnlich beginnen Metallica-Konzerte seit 1983 mit dem sich stetig dynamisch steigernden Ennio Morricone-Stück »Ecstasy of Gold« aus dem 
Western The Good, the Bad and the Ugly. Auf dem Höhepunkt hat die Band unbemerkt die noch dunkle Bühne betreten und beginnt in der Regel ohne Ansage mit einem Hit, meist begleitet von pyrotechnischen Effekten und Light Show. Nicht so auf dieser Tournee: Ohne jegliche akustische oder visuelle Ankündigung und ohne, dass die Hallenbeleuchtung ausgeschaltet würde, laufen die Bandmitglieder durch den Security-Graben zwischen Bühne und Publikum und klatschen die ausgestreckten Hände der Fans ab. Im Konzertfilm trinkt Drummer Lars Ulrich dabei aus einem Plastikbecher, spuckt sein Getränk mehrfach den Fans entgegen und reicht den Becher dann ins Publikum. Dies demonstriert Nähe zu den Fans, zeigt, dass die Band keinerlei Berührungsängste hat. Das Teilen der Nahrung mag man als Symbol vertraulicher Gemeinschaft deuten, das Anspucken signalisiert die Band und Fans verbindende Absage an bürgerliche oder elterliche BenimmKonventionen. Auf der Bühne angekommen, hängen die Musiker ihre Instrumente um und schlagen freundschaftlich die Fäuste gegeneinander, offenbar, um sich viel Erfolg zu wünschen, was deutlich macht, dass sie nicht abgeklärt professionell agieren, sondern auch ihnen nun etwas Aufregendes bevorsteht. Der Kleidung der Musiker sieht man nicht an, dass sie vielfache Millionäre sind. Im Gegenteil: der Bassist trägt wie die meisten Zuschauer ein Tour-T-Shirt der Band, Ulrich zeigt sich während des gesamten Konzerts intim wie unter Freunden: in Boxershorts und Unterhemd, später mit freiem Oberkörper. Dieses Outfit erscheint rein funktional und kündigt die zu erwartende körperliche Verausgabung an. Die Musiker geben sich offen und unprätentiös, heben sich nicht als Stars $a b$ - und bringen damit persönliche und sozio-kulturelle Authentizität zum Ausdruck. Ulrich wirft noch Drumsticks in die Menge, bevor die Band beginnt, für etwa 90 Sekunden zu jammen. Erneut verlässt Ulrich sein Schlagzeug, um Kontakt mit dem Publikum zu suchen. Frontman James Hetfield beginnt dann den Titel »So What «, eine B-Seite der britischen Punk-Band Anti-Nowhere Leaugue aus dem Jahr 1981, den Metallica 1993 ebenfalls nur auf einer Single-Rückseite veröffentlicht hatten - eine Geste für die »echten« Kenner, nicht für die Laufkundschaft. Erst spät im Verlauf des Stückes wird die Hallenbeleuchtung ausgeschaltet und die Light Show setzt ein. Die Bühne steht dabei in der Hallenmitte und ist von allen Seiten einsehbar. Selbst Backstage-Aktivitäten können vom Publikum verfolgt werden, was die handwerkliche Authentizität unter Beweis stellen soll: die Band hat nichts zu verbergen, nirgendwo sind Synthesizer, zusätzliche Musiker oder Playback-Abspielgeräte versteckt.

Insgesamt ist diese Eröffnung geprägt von Understatement und dem Bemühen um Natürlichkeit und Spontaneität. Sie ist als demonstrative Absage an die Konvention zu verstehen, das Publikum gleich zu Beginn mit einer 
sorgsam inszenierten Überraschung, spektakulären Licht-Effekten und einem großen Hit zu überwältigen. Stattdessen versucht man deutlich zu machen, dass keine ablenkende Show zu erwarten ist, sondern »the real thing « (was sich allerdings noch als unzutreffend herausstellen wird). Alles soll so nichtinszeniert wie möglich wirken - und gerade dies folgt eindeutig einer Inszenierungsstrategie, die vor dem Hintergrund der Ausverkaufs-Vorwürfe zu verstehen ist, mit denen sich die Band in den 1990er Jahren angesichts des großen Mainstream-Erfolgs und des korrespondierenden stilistischen Wandels immer wieder konfrontiert sah.

Weitere Strategien zur Demonstration von Authentizität lassen sich am Beispiel der Toten Hosen zeigen. Zu jedem Konzert gehört hier in alter Punk-Tradition das Stage Diving des Sängers Campino, an dem sich manchmal auch der Bassist - samt Instrument - beteiligt. Das Bad in der Menge führt vor, dass unmittelbarer körperlicher Kontakt und Kommunikation mit den Fans gesucht werden. Zum anderen schafft es einen Moment der Spontaneität, da unvorhersehbar ist, wann, wo und in welchem Zustand das Publikum den Sänger wieder auf die Bühne entlässt. Das gilt noch gesteigert, wenn diesem Besuch ein riskanter Sprung vom Dach der Bühnenkonstruktion vorausgeht, der Sänger also darauf vertraut, dass das Publikum inn sicher auffängt. Campino ist sich des Symbolcharakters des Stage Divings im Sinne der sozio-kulturellen Authentizität durchaus bewusst:

»Ich glaub, das is auch das, was die an uns gut finden. Also... die schätzen das sehr, wenn man sich nich für irgendwas zu schade ist, ja? Und diesen bedingungslosen Einsatz, den liefern wir denen ja auch. Was soll denn das anderes sein, wenn man sich ins Publikum schmeißt von der Bühne! Was soll das sein? Das ist doch nur 'ne Geste, 'ne hilflose Geste, um den Leuten zu zeigen: trotz dieser Barriere und dem Graben sind wir irgendwo auf Augenhöhe mit euch« (in Kablitz-Post 2009 bei 19:00).

In diesem Sinne können auch die verhältnismäßig moderaten und nicht nach Platzqualität gestaffelten Ticketpreise der Toten Hosen als Geste der soziokulturellen Authentizität an die Fans verstanden werden. ${ }^{6}$

Spielfehler oder Textausfälle werden bei ihren Konzerten nicht übergangen, sondern bewusst herausgestellt und kommentiert. So bricht die Band den Song »Paradies « am 3.7.2009 in Berlin zu Beginn der zweiten Strophe ab: »Ich muss zugeben, dass ihr die Texte allemale besser drauf habt als ich. Ich finde, der Rest des Publikums hat eine ordentliche Version verdient! Wer hat die zweite Strophe drauf? Probier's, komm rauf!« (s. Die Toten

6 Einheitlich ca. 40 Euro verlangten die Toten Hosen im Jahr 2012, während für Konzerte von Herbert Grönemeyer 60 Euro, von Westernhagen 71 bis 95 Euro zu zahlen waren (Recherche bei eventim.de und ticketmaster.de am 21.8.2012). 
Hosen 2009). Ein Fan wird auf die Bühne geholt und vorgestellt, lachend beginnt die Band neu und überlässt ihm den Gesang. Dies stellt den LivenessCharakter deutlich heraus, demonstriert im Scheitern immerhin handwerkliche Authentizität und wirkt offen, ehrlich und spontan. Ist es aber nicht: Exakt an derselben Stelle wurden auf dieser Tour auch die Konzerte in Langenselbold, Losheim, Ludwigsburg etc. mit der gleichen Aktion unterbrochen, wie eine kurze YouTube-Recherche offenlegt. Nichtsdestotrotz schaffen solche Inszenierungen Raum für unvorhersehbare Interaktionen und betonen, dass es Band und Fans weniger um den perfekten Vortrag als um das einmalige gemeinsame Erlebnis geht. Mit derselben Absicht werden auf die Bühne geworfene Gegenstände sofort kommentiert, Songs auf Zuruf gespielt oder bestimmten Mitgliedern im Publikum gewidmet. Ebenfalls als Hinweis auf Authentizität ist die körperliche Verausgabung der Musiker zu deuten: Schweiß - wie bei Metallica auf nackten Oberkörpern - lügt nicht, er signalisiert, dass die Musiker sich nicht schonen und unter vollem Körpereinsatz hart für ihr Publikum arbeiten, statt nur ein Pflichtprogramm zu absolvieren. Notfalls werden zweistündige Auftritte auch mit frischem Kreuzbandriss (bei Rock am Ring 2000) oder mit eingegipstem Fuß und Krücken durchgestanden - ohne dass deshalb auf den Sprung in den Zuschauerraum verzichtet würde (bei Rock am Ring 2008, s. Die Toten Hosen 2008).

Als Zeugnis von persönlicher Authentizität wird der Ausdruck von Spielfreude und Spaß auf der Bühne gedeutet, den die Musiker durch Gesten und Kommunikation untereinander signalisieren können. Besonders authentisch wirkt es, wenn die Fans den Eindruck gewinnen, die Band würde unabhängig von der Bezahlung (nur an diesem Abend!) besonders lange spielen, weil sie selbst so großen Spaß daran hat. Dass Stücke, die seit vielen Jahren allabendlich gespielt werden, der Band trotzdem persönlich noch etwas bedeuten, versucht Campino durch Ansagen, die sie autobiographisch verorten, deutlich zu machen. Gecoverte Songs dienen weniger der Reminiszenz an die Anfangstage, sondern weisen die Band als Fans und als Kenner aus, die sich nicht zu schade sind, ihren Vorbildern die Referenz zu erweisen. Zudem verorten Coverversionen die Gruppe in einer bestimmten Tradition. So spielen die Toten Hosen nicht selten Songs von The Clash und den Ramones, was sie in der Punk-Tradition erdet, oder aktuell von Hannes Wader, um einen Bezug zum sozialkritischen, linken Liedermachertum herzustellen (so bei Rock am Ring 2012). 


\section{Strategien der Verkleinerung}

Je größer mit zunehmender Popularität die Bühnen und die Zuschauerräume werden, umso dicker muss aufgetragen werden, um den Liveness-Charakter und den Eindruck von Nähe performativ herzustellen. Die dazu in der Regel eingesetzten Großbildleinwände machen zwar auch die kleinste Geste in der letzten Reihe sichtbar, drohen dabei aber eher den Eindruck des passiven Fernsehguckens zu vermitteln als den des Live-dabei-Seins. Da man auch den Zuschauern auf den hinteren Plätzen eines 60.000er-Stadions ein visuelles Spektakel bieten will, werden spezielle Licht-Arrangements, Pyrotechnik, aufblasbare Puppen usw. eingesetzt, die von Computerprogrammen gesteuert werden und dadurch Spontaneität im Konzertablauf oder während der Songs verhindern. Durch die notwendige Höhe der Bühne und die aus Gründen der Sicherheit erforderliche große Distanz zwischen Zuschauerraum und Bühne sind die Möglichkeiten der Interaktion von Aufführenden und Zuschauern stark eingeschränkt. Seit den 1990er Jahren sind daher zwei weitere Konzepte der Inszenierung von Liveness und Authentizität zu beobachten, die dieser Entwicklung entgegenwirken sollen: Zum einen das paradoxe MTV-Unplugged-Szenario, das - vor dem Fernseher - ein »authentischeres « Live-Erlebnis verspricht als es das Stadionkonzert bieten kann (s. Auslander 1999: 96-111). Es setzt auf eine Verkleinerung der Bühne und des Zuschauerraumes, um so - beobachtet von zahllosen Kameras, ausgeleuchtet von unzähligen Scheinwerfern und mit nicht weniger Kabeln und Steckern als jedes andere Konzert - Nähe und Intimität herzustellen. Neben diesem »intimeren« Rahmen eines Fernsehstudios werden im UnpluggedSzenario vor allem drei Strategien zur Suggestion resp. Präsentation von Authentizität eingesetzt: Auf das visuelle Spektakel des Stadion-Konzertes kann verzichtet werden, manchmal ist sogar die Lightshow »unplugged «, wenn man den Einsatz von Kerzen auf der Bühne so deuten will. Ohne visuelle Ablenkungen muss die Band das Publikum nur mit ihren musikalischen Fähigkeiten überzeugen. Zweitens soll durch den vorgeblichen Verzicht auf elektronisch bearbeitetes und verstärktes Instrumentarium auch die Musik direkter, »ehrlicher « im Sinne der emotionalen Authentizität wirken. Indem angeblich technische Hilfen wegfallen, soll sich in der »handgemachten« Musik der »wahre Kern« zeigen, zudem kann die handwerkliche Authentizität so besser vorgeführt werden. Drittens besteht das Repertoire der Unplugged-Konzerte zum großen Teil aus ruhigen Balladen, die sich zur Herausstellung emotionaler Authentizität vermeintlich besser eignen als laute und schnelle Stücke, zumal auch der Glaube herrscht, emotionaler 
Ausdruck könne ohne technische Mittler unverstellt, also intensiver und »echter« vermittelt werden.

Das zweite Inszenierungskonzept, um den Liveness-Charakter von Konzerten im Zeitalter der großen Stadien-Events zu stärken, ist die B-Stage: eine sehr viel kleinere, von allen Seiten einsehbare Bühne in der Mitte des Stadions, die die Band nach zwei Dritteln des Konzertes händeschüttelnd über einen Catwalk erreicht, um das Konzert dort mit reduzierter Lichtshow für drei Stücke fortzusetzen. Gern wird diese Reduzierung des Bühnenraums mit dem Unplugged-Konzept verbunden und es werden dort Balladen mit Akustik-Gitarren gespielt (s. Coldplay 2009). Alternativ kann die B-Stage aber auch genutzt werden, um zu demonstrieren, dass die Band ihren Wurzeln treu bleibt und sich trotz des großen Erfolges im Kern nicht verändert hat: Gespielt werden dann die ersten Hits oder - wie damals in der Garage - Coverversionen (so nicht selten bei den Rolling Stones).

Eine perfide inszenierte Variante der B-Stage nutzten auch Metallica auf der oben beschriebenen Tournee: Am Ende des Songs »Enter Sandman« scheint ein Beleuchter in der oberen Bühnenkonstruktion während eines Pyro-Effektes plötzlich seinen Halt zu verlieren, schwingt kopfüber an einem Sicherungsseil über der Bühne und stürzt dann ab. Zahlreiche weitere Explosionen ereignen sich, diesmal anscheinend ungeplant. Sicherheitskräfte eilen auf die Bühne, der Sänger wird scheinbar von einem Gegenstand getroffen, hält sich den Kopf und geht zu Boden. Die Band hört auf zu spielen, über die PA zischt und brummt es chaotisch, weitere Beleuchter fallen von oben, ein Roadie rennt in Flammen über die Bühne. Zunächst sieht man noch einen Notarzt, Feuerlöscher, Taschenlampen, Wiederbelebungsversuche, zuckende Scheinwerfer und den Zusammenbruch eines Teils der Bühnenkonstruktion, dann verhüllt Qualm die Sicht. Kurz darauf betreten die Bandmitglieder wieder die Bühne und geben vor, überrascht zu sein (»What the fuck happened?«). Kleine Combo-Verstärker werden vermeintlich spontan auf die Bühne getragen, statt der zerstörten Lichtanlage sorgen von der Decke herabgelassene nackte Glühbirnen für minimale Beleuchtung. Nachdem kurz mit einem Jam das neue Equipment getestet wurde, spielt die Band in diesem reduzierten Setting - der B-Stage-Konvention entsprechend - eine Coverversion und einen Song vom Debutalbum. Damit knüpft sie an ihre verschiedentlichen Versuche an, sich trotz des Millionen-Umsatzes als einfache Garagen-Band darzustellen (etwa auf der »Garage Days Revisited « überschriebenen B-Seite der Maxi-Single Creeping Death (1984), der EP The \$5.98 E.P.: Garage Days Re-Revisited (1987) und der Doppel-CD Garage Inc. (1998), die alle ausschließlich Cover-Versionen beinhalten). Wie ein Makingof auf der DVD Cunning Stunts deutlich macht, wurde dieser Ablauf minutiös 
durchgeplant, gründlich geprobt und allabendlich wiederholt. Dennoch hielten die meisten Zuschauer den inszenierten Unfall in der Live-Situation für echt, lokale Radiosender berichteten am nächsten Tag über Verletzte. Zumindest das Ausmaß der Inszenierung blieb im Unklaren, da sich einige Beteiligte an einzelnen Abenden tatsächlich ernsthaft verletzten.

Wem das Unplugged-Szenario zu klischeehaft inszeniert erscheint, der sei auf das US-amerikanische National Public Radio verwiesen, wo im Rahmen der »Tiny Desk Concerts«-Reihe (http: //www.npr.org/series/tiny-deskconcerts) regelmäßig Bands ins Redaktionsbüro eingeladen werden, um dort ohne Bühne vor der Belegschaft und zwei Kameras zwar nicht unplugged, aber doch unter Verzicht auf jede Art von Bühnentechnik ihre Live-Qualitäten zu demonstrieren. Für ein Schlagzeug ist dort kein Raum, sodass (so im Falle des Wilco-Auftritts) auch gewöhnliche Schreibtisch-Utensilien als Percussion-Instrumente herhalten müssen. Es geht noch extremer: Für die englische Reihe »Black Cab Sessions« (http://www.blackcabsessions.com) werden Musiker gebeten, einen Song während einer Taxi-Fahrt durch London zu spielen. Stärker kann man den Bühnenraum nicht beschränken, was leider auch das Live-Publikum auf den Taxi-Fahrer und den Kameramann reduziert. Auch bei diesen beiden Formaten handelt es sich in dem Sinne um Inszenierungen, als bewusst Bedingungen geschaffen werden, unter denen tatsächliches Sein statt täuschendem Schein zum Vorschein gebracht werden soll - indem die Musiker nämlich zeigen können, wie viel von ihrem Reiz übrig bleibt, wenn man ihnen nur zwei Kubikmeter, einen Schellenkranz und eine Akustikgitarre zur Verfügung stellt. Diese Auftritte werden im Internet veröffentlicht und dienen so vor allem Musikern aus dem »Independent «-Bereich als besonders glaubwürdige Promotion-Instrumente. Wie viele Fehlversuche es neben dem veröffentlichten Clip gibt, erfährt das Publikum dabei natürlich nicht.

Eine eigene Variante der Verkleinerungs-Strategie haben auch die Toten Hosen entwickelt. Abseits größerer Tourneen treten sie auch nach 15 Millionen verkauften Alben gelegentlich - »wie früher « - in den Wohnzimmern oder Partykellern von Fans auf, die sich um die Ausrichtung solcher Konzerte bewerben können. Die Band verzichtet bei diesen Anlässen auf Gage, spielt gegen Kost und Logis beim Gastgeber und zahlt die Anreise selbst. Zum 30-jährigen Bühnenjubiläum wurde 2012 eine Tour mit sechzehn solcher Auftritte unternommen. Bei allen Aufenthalten wurde vor, während und nach den Shows mit mehreren Kameras gefilmt, sodass sich jede Station - inklusive Bildern vom Aufwachen der Musiker mit zerzausten Haaren auf dem Fußboden einer fremden WG - auf der Homepage dokumentiert findet (s. Die Toten Hosen 2012). Jeder kann sich so von der persönlichen, sozio- 
kulturellen und handwerklichen Authentizität der Musiker überzeugen und die Band zugleich medienwirksam für ein neues Album werben - ein Marketing, das die Band »so herzhaft« betreibe, »dass es nicht negativ auffällt«, wie die Süddeutsche Zeitung findet (Arnu 2012: 9). Auch das ZDF ist sich sicher: "Dass diese Tour keine Beweisshow ihrer Fantreue ist, sondern immer noch echter Jungens-Spaß, das spürt man in jedem Moment« (ZDF Aspekte 2012 bei 6:40).

\section{Die Inszenierung authentischer Inauthentizität}

Neben all diesen mal mehr, mal weniger offensichtlichen Bemühungen, die Inszenierung von Authentizität nicht auffällig werden zu lassen, gibt es schon seit den frühen 1970er Jahren auch die Gegenoffensive: das Bestreben, eine so offensichtlich inszenierte Bühnenshow zu bieten, dass niemand auf die Idee kommen kann, hier sei etwas Privates, Unverstelltes über die Musiker zu erfahren. Diese postmoderne Tradition reicht von David Bowies theatraler Ziggy Stardust-Tournee (s. Jooß-Bernau 2010: 240ff.) über Roxy Music, die 1972 mit exaltierten Kostümen, ironischer Bühnenshow sowie herausgestellter Künstlichkeit die Rock-Authentizität ihrer Zeit parodierten (s. Pattie 2007: 84f. sowie Roxy Music 1972 u. 1973), über die Pet Shop Boys und U2 (Zoo-TV- bzw. Zooropa- und Popmart-Tourneen, s. Jost 2011) bis zu Madonna und Lady Gaga. Sie alle haben den Inszenierungscharakter ihrer Konzerte deutlich herausgestellt und sich damit zugleich der konventionellen Inszenierung von Authentizität verweigert.

Als Beispiel möchte ich die Pet Shop Boys herausgreifen, die sich 1981 gründeten, bis 1989 aber nicht auf Tournee gingen (»It's kinda macho nowadays to prove you can cut it live. I quite like proving we can't cut it live. We're a pop group, not a rock and roll group «, so Neil Tennant in Goodwin 1988: 44). Als sie dann doch live auftraten, kehrten sie alle bisher vorgestellten Marker von Authentizität um. Was bei ihren Konzerten live gespielt und was vorprogrammiert ist, bleibt stets im Unklaren: Zum Gesang Tennants spielt Chris Lowe an seinen Keyboards offenbar einzelne Melodien und Akkorde, der Großteil der Backing Tracks wird aber von verschiedenen MIDIgesteuerten Sequenzern abgespielt (»Call it performance / Call it art / I call it desaster / If the tapes don't start « heißt es selbstironisch in ihrem Song »Electricity«). Improvisierte Momente oder spontane Variationen sind so weder möglich noch beabsichtigt. Gesten der Emotionalität, Jammen, StageDiving, Kumpanei mit dem Publikum, Schweiß und nackte Oberkörper wird man hier nicht zu sehen bekommen, stattdessen gibt es zahlreiche synchronisierte Videosequenzen und aufwändige Choreographien mit mehreren Tän- 
zern. Zu Beginn der Konzerte ihrer Pandemonium-Tour (2009/10) waren die Köpfe der Musiker mit weißen Würfeln maskiert, im weiteren Verlauf trugen sie dunkle Anzüge mit Melone, militärische Wintermäntel, Ski-Jacken und violette Fellmützen oder Königskronen, während die Tänzer in Weihnachtsbaum- oder Wolkenkratzer-Kostümen auftraten. Rein gar nichts in dieser durchinszenierten Show bemüht sich, persönliche, sozio-kulturelle, emotionale oder handwerkliche Authentizität zu suggerieren. Statt wie sonst üblich die kreative Arbeitsteiligkeit herunterzuspielen, reklamiert die Band nicht einmal die Autorschaft für all diese visuellen Aspekte der Show: »Ehrlich gesagt, entstehen Dinge oft, ohne dass wir selbst wirklich etwas dazu beitragen« (Tennant in Bönisch 2009: 9).

Der von Grossberg (1992: 226) geprägte Begriff der »authentic inauthenticity« will zum Ausdruck bringen, dass Musiker wie die Pet Shop Boys gerade durch ihre zur Schau gestellte Ablehnung aller Authentizitätsbemühungen als umso authentischer angesehen werden können. Ihr Auftreten wird als ideologiekritische Absage an die allgegenwärtige Inszenierung von Authentizität aufgefasst. Dies betrifft sogar die verweigerte emotionale Authentizität: »In [Tennant's] flat, regular delivery, especially when this is combined with his generally static posture, the refusal of emotional involvement he conveys is widely perceived as a refusal to >cheat < the listener « (Moore 2002: 214). Authentizität bleibt damit auch in diesem Fall ein wichtiges Kriterium: Weil sie eine klare Position beziehen, die sie konsequent vertreten, und weil sie eine realistische statt einer romantisierten Weltsicht zum Ausdruck bringen, gelten die Pet Shop Boys ihren Fans sicher nicht als inauthentisch. In den konservativen Kritiker-Kanon, wie er z.B. wirkmächtig vom Rolling Stone vertreten wird, haben es die genannten Bands mit dieser Haltung freilich bis heute nicht geschafft (s. Appen/Doehring 2006).

Während bei den genannten authentisch-inauthentischen Musikern noch eine Kritik an der Authentizitäts-Ideologie zu erkennen ist, gibt es und gab es immer schon auch Musiker und Musikrichtungen, für deren Anhänger die Betonung von Authentizität und Liveness auf der Bühne offenbar nur eine geringe oder überhaupt keine Rolle spielen. So können die Black Eyed Peas (2011) oder Kanye West (2012) ohne Instrumente auf der Bühne auftreten und dabei Auto-Tune zur Stimmverfremdung nutzen, ohne dass sich ihr Publikum daran stören würde. Katy Perry bringt es während ihres Songs »Hot N Cold« im Rahmen der California Dreams-Tour auf sechs Kostümwechsel in vier Minuten (s. Perry 2011). Ihre Fans kritisieren die durchchoreographierte Show, bei der jede Bewegung auf der Bühne vorgegeben ist, keineswegs als über-inszeniert und inauthentisch: 2011 wurde Perry bei den Teen Choice 
Awards und den MTV Europe Music Awards als »Best Live Act« ausgezeichnet.

Schaut man sich jedoch die Listen der kommerziell erfolgreichsten Tourneen der Jahre 2010 und 2011 an, wie sie von Billboard und Pollstar erhoben wurden, so dominieren dort mit Bon Jovi, AC/DC, U2, Taylor Swift, Metallica sowie James Taylor \& Carol King Musiker, die nach wie vor auf die demonstrierten Marker von Authentizität setzen (vgl. Reinartz 2010, Smith 2011, Billboard 2010 u. 2011). Zumindest beim zahlungskräftigen und damit vermutlich älteren Publikum gibt es offensichtlich nach wie vor ein ungebrochenes Verlangen nach dieser Form von Authentizität.

\section{Adele Live}

Die Einschätzung, dass die generelle Wertschätzung von Authentizität auch im 21. Jahrhundert keineswegs passé oder nur eine Sache der männlich dominierten Rock-Ästhetik ist, möchte ich abschließend durch einen Blick auf die Inszenierung von Adele bekräftigen, der kommerziell weltweit erfolgreichsten Musikerin des frühen 21. Jahrhunderts.

Auf ihrer jüngsten Tournee suggeriert schon die Bühnendekoration Intimität und eine Absage an konventionelle Hi-Tech-Show-Inszenierungen. Die gewöhnlichen Lampenschirme, in deren Schein sie auftritt, Blumenarrangements und der Verzicht auf eine Video-Wand sollen für eine private Wohnzimmer-Atmosphäre sorgen, so als ob die Sängerin ihr Publikum zu sich nach Hause eingeladen hätte. Dieses Herunterspielen der Distanz von Star und Publikum ist ein zentrales Element ihres Auftretens; immer wieder bemüht sie sich, den Eindruck zu erwecken, als wäre sie die beste Freundin oder »the girl next door «, keine Multimillionärin. So zeigt sie sich im Making-of ihrer Live at the Royal Albert Hall-DVD am Morgen des Konzertes bodenständig in der Küche ihrer eigenen Wohnung, wo sie sich äußerst staruntypisch im Morgenmantel und mit Lockenwicklern einen Instant-Tee aufgießt, umkreist von ihrem Dackel. „I wanna make them feel like they know me. They do know me, but I want 'em to feel like we're just here and that we're just having a cup of tea and a take-away or something like that on a Saturday night «, erklärt sie, während die Kamera über ihr Bett und ihre Bücherregale schweift (0:20-0:30). Später, in der Maske vor dem Konzert, macht sie deutlich, dass es bei ihr keine Maske gibt: „It's not a persona because it's completely me but it's like... I'm a bit more of, like, kind of to myself and shy in a way, like normally. I don't know what possesses me to chat so much shit on stage, I really don't« (2:47-3:06). Dies tut sie in der Tat: Auf der Bühne verhält sie sich so offenherzig, als würde sie alle Zu- 
schauer persönlich kennen und als unterhielte sie sich mit ihnen im Pub. Sie lacht dabei laut über ihre eigenen Witzchen, prustet ins Mikrophon und bezeichnet sich selbst als »chatterbox«. Ihre Aufgeregtheit zu Beginn des Konzertes versucht sie nicht zu verbergen, sondern sie verrät dem Publikum:

»I'm still shitting myself. I haven't settled down yet. I'm not sure if I'm gonna, actually. I'll probably go home, going home on my own to my empty flat, I'll probably always be buzzing [unverständliches Gemurmel] and I don't know what to do with myself« (Adele: Live at the Royal Albert Hall, 25:53).

Adele spricht in breitem Cockney darüber, wie sie selbst als Fan die Spice Girls in der Royal Albert Hall bewundert hat und kommentiert das Ambiente mit den Augen eines begeisterten Fans: »It's so posh here, isn't it?« (1:07:12). Sie trinkt zwischen den Songs Honig-Milch aus einem Becher mit Dackel-Motiv, winkt immer wieder ins Publikum, outet sich als »Sex and the City«-Zuschauerin und plaudert von ihrem Friseur-Besuch am Vortag, nicht ohne den Anwesenden einen Bericht weiterzuempfehlen, den sie dort in einer Illustrierten gelesen habe. Sie begrüßt ihre Freundinnen im Publikum und erzählt detailreich Geschichten aus dem gemeinsamen Privatleben, die diese, im Publikum von den Kameras eingefangen, nickend mit einem Lachen bestätigen (31:32).

Vor allem aber verortet Adele in ihren Ansagen den Ursprung ihrer Songs in autobiographischen Erfahrungen. Immer wieder erzählt sie von der Beziehung, auf deren schmerzvollem Scheitern fast alle Songs des aktuellen Albums 21 basierten und behauptet, immer noch bei jedem Auftritt an die jeweiligen Erlebnisse zu denken: "When I'm singin' my songs I vision the person, I vision my ex who this song is about - like all the other songs and I vision him and I sing them out to him « (52:19). Dabei lässt sie einfließen, dass sie die Songs selbst geschrieben habe, wobei ihre tatsächliche Rolle nicht ganz klar ist, da in den Liner Notes diverse Mitkomponisten genannt werden. Ganz im Sinne des McCarthy-Zitats zur emotionalen Authentizität (s. S. 45f.) erklärt Adele, sie erkenne sich erst in ihren Songs selbst:

»This song just sort of came out of me and I was really surprised [about], you know, the contents of this song 'cause I never know how I feel, I never let myself know how I feel. I kinda put it to the back of my mind and I only find out what I'm thinking and what I'm feeling in my songs, but I didn't realize I was feeling like this« (37:00).

Dass ihre Stücke ihr persönlich emotional nahe gehen, lässt sie nicht nur in ihren Ansagen wissen (»Always makes me sad that one, doesn't matter where or when I sing it «, 42:46): Für »Someone Like You« wird Intimität suggeriert, indem das Licht gedämpft wird und alle Mitmusiker bis auf den 
Pianisten backstage bleiben. Es gibt keine ablenkende Show, nur volle Konzentration auf den melismen- und rubatoreichen Gesang, den sie mit erstem Blick oder geschlossenen Augen vorträgt. Sie beginnt einige Phrasen bewusst verfrüht, hält viele Töne lang aus, phrasiert jede Zeile individuell und bemüht sich auch in Mimik und Gestik ostentativ um einen möglichst intensiven emotionalen Ausdruck. Am Ende des Stückes bricht sie in Tränen aus, überwältigt von ihrem eigenen Song und den Standing Ovations des Publikums, in dem die Kameras ebenfalls tränenreiche Gesichter entdecken. Adele hat sofort ein Taschentuch zur Hand, wendet sich nicht ab, sondern bringt ihre Gefühle sogleich wieder zur Sprache: »That song makes me sad anyway and it takes my breath when you sing it like that back to me [...] it's all a bit much $\ll(1: 32: 32)$.

Als der Zugabenteil naht, nutzt sie dies wiederum für eine Absage an Show-Konventionen und zur Demonstration ihrer Ehrlichkeit:

»This next one that I'm gonna do for you is gonna be my last song tonight [Buh-Rufe]. I'm joking! I'm adding some drama to my show! I don't dance, ain't no fire, ain't no greased up men nowhere [...]. Anyway, now I'm just gonna pop offstage and pretend that I'm not coming back. But I will! I won't be gone very long, I normally just gonna take my shoes off and take a sip of water, then [unverständlich] back on. [...] I really don't enjoy encores, by the way. I think unless you're Dylan, or Paul Simon or Madonna or something like that, you should stay on the fucking stage [Gelächter]. I don't have a catalogue of music yet. When I do, I'll keep you waiting for sure« (1:16:001:16:40 bzw. 1:23:15).

Wenig überraschend ist es da, dass es auch bei Adeles Konzerten einen reduzierten »Unplugged «-Part nebst Cover-Version gibt, für den die Musiker im Halbkreis in der Bühnenmitte zusammenrücken. Und auch ein Liveness und handwerkliche Authentizität verifizierender Fehlstart wurde aus dem Konzertfilm bewusst nicht herausgeschnitten (»That was a shit note, let's start again«, 38:40).

Das Publikum ist überzeugt und begeistert von Adeles Offenheit, Ehrlichkeit und Natürlichkeit, wie kurze Fan-Statements im Making-of deutlich machen sollen:

»It touches a part in you, doesn't it? It's honest and it's credible« (2:14).

»Beautiful love songs, right from the heart« $(6: 54)$.

"She talks the way she wants to talk, she says the things she wants to say" (6:58).

Neben den regulären Konzerten hat Adele zur Promotion des Albums 21 zahlreiche Radiosender, darunter auch NPRs »Tiny Desk Concerts«, besucht 
und dort mit Minimal-Besetzung (meist von Kameras begleitet) live gesungen, um zu zeigen, dass sie keine visuelle Inszenierung und keinen technischen Aufwand braucht, dass ihre Stimme »echt « ist und sich in jeder LiveSituation bewährt. Durch diese Betonung ihrer handwerklichen Authentizität und das Herunterspielen visueller Inszenierungen setzt sie sich dezidiert von kostümierten und stark sexualisierten Konkurrentinnen ab, wie sie in einem Rolling Stone-Interview deutlich macht:

»Even if I had a really good figure, I don't think I'd get my tits and ass out for no one. I love seeing Lady Gaga's boobs and bum. I love seeing Katy Perry's boobs and bum. Love it. But that's not what my music is about. I don't make music for eyes, I make music for ears « (in Touré 2011). ${ }^{7}$

\section{Hintergründe}

In nahezu allen Geisteswissenschaften wird Authentizität aktuell umfangreich diskutiert; das Spektrum relevanter Felder reicht dabei von Politik, Religion und Geschichtsschreibung über Fremdsprachenunterricht, Werbung, Tourismus, Lebensmittel, Trachtenmode und Pornographie bis zu bildender Kunst, Theater, Film, Reality-TV, Literatur und eben Musik (im Überblick: Funk/Krämer 2011, Vannini/Williams 2009, Amrein 2009, Lindholm 2008). Obwohl Wissenschaftler sich schon länger um ihre Dekonstruktion bemühen (so z.B. Frith 1987, Weinstein 1999), beobachten Michael Rössner und Heidemarie Uhl (2012) gegenwärtig eine »Renaissance der Authentizität«, Charles Taylor (2007: 473) charakterisiert unser Zeitalter gar als »age of authenticity«.

Ohne Zweifel kommt in der ubiquitären Suche nach Authentizität ein zentrales Bedürfnis unserer Zeit zum Ausdruck. Das gestiegene Verlangen nach Echtem, Natürlichem, Unmittelbarem, Glaubwürdigem wird oft mit dem Verweis auf tief greifende gesellschaftliche, ökonomische und technologische Veränderungsprozesse zu erklären versucht, die mit dem schwammigen Etikett der Postmoderne eher hilflos und unscharf als tatsächlich erhellend zusammengefasst werden. Drei Ansatzpunkte möchte ich zum Abschluss thesenartig herausgreifen, die ein weiteres Nachdenken über die Hintergründe des Verlangens nach Authentizität anregen können:

7 Dieses authentifizierende Understatement zeigt sich übrigens bereits in der Benennung ihrer Alben 19 und 21 (was jeweils ihrem Alter bei den Aufnahmen entspricht) und ihren Tourneen, die »Adele live« und »An Evening with Adele betitelt waren. Adele ist zudem ihr echter Vorname, was wiederum Natürlichkeit und Offenheit signalisiert - im Unterschied etwa zu Lady Gaga. 
1. Selbstverwirklichung und persönliche Authentizität sind zwar bereits seit der Aufklärung und insbesondere der Romantik wesentliche Werte der westlichen Kultur; als »Ideal einer radikal individualisierten Lebensführung « haben sie Christoph Menke (2005: 309) zufolge aber ausgehend von den Jugend- und Studentenbewegungen um 1968 und »mit deren Entpolitisierung zu einer Kultur des individualistischen Hedonismus« einen Boom erfahren. Den Wunsch, sich nicht verbiegen zu müssen und ein selbstbestimmtes Leben zu führen, können jedoch nur die wenigsten tatsächlich verwirklichen. Frei tätige Künstler, heute wohl vor allem erfolgreiche Musiker, dienen daher als beneidenswerte Vorbilder und als Projektionsfläche für das Ideal eines nicht-entfremdeten, »authentischen Lebens « - denn dass sie ein solches führen, bemühen sich Musik- und Boulevardpresse sowie (Auto-) Biographik nach Kräften zu vermitteln. Auch gegen besseres Wissen mögen viele den Glauben nicht aufgeben, dass trotz der beherrschenden Logik des Kapitals dort noch ein selbstverwirklichendes Leben möglich ist, das sich den Regeln der Ökonomie verweigert (vgl. Chiapello 2010: 38f.). Das Perfide daran: Die Kulturindustrie unterstützt diesen Glauben gern, denn Authentizität schafft Umsatz.

2. Musik dient der Identitätsarbeit und der sozialen Distinktion, sie zeigt uns und anderen, was für Menschen wir sind (vgl. Kotarba 2009). Je stärker Musik diese sozialpsychologischen Funktionen erfüllt, desto wichtiger wird das Kriterium der Authentizität für ihre Bewertung. Denn wer sich über seinen Musikgeschmack identifiziert, der wird gründlich prüfen, ob die Musiker tatsächlich für die Wertegemeinschaft stehen, die sie zu repräsentieren vorgeben, und ob das Bekenntnis zu ihnen wirklich einen Distinktionsgewinn verspricht - andernfalls drohen Blamage und die verunsichernde Infragestellung der eigenen Position. Besonders erfüllt Musik diese Funktionen entwicklungsbedingt in der Jugend, daneben aber auch in Teilkulturen, die sich aufgrund ihrer Ethnizität, sexuellen Orientierung oder politischen Überzeugungen nicht mit der Mehrheitskultur identifizieren.

Dass die Arbeit an einer stabilen Identität seit einigen Jahrzehnten zu einer immer größeren Herausforderung geworden ist, stellen viele Soziologen und Psychologen einhellig fest. "Identität, so scheint es, wird in Alltag und Wissenschaft zum Dauerthema, weil die tradierten gesellschaftlichen und kulturellen Grundlagen für eine stabile soziale Verortung und Einbindung der Menschen zunehmend wegbrechen«, so Rolf Eickelpasch und Claudia Rademacher (2004: 5).

»Dem Gewinn an Wahlmöglichkeiten und Optionsspielräumen für die Ausgestaltung des >eigenen Lebens`steht ein Verlust kollektiver Sicherheit und 
Zugehörigkeit gegenüber. Die gesellschaftliche Forderung, sich aus vorgefertigten Fragmenten und Versatzstücken eine eigene Biografie und eine eigene Identität zu konstruieren, stellt für den Einzelnen ein anstrengendes, störungsanfälliges, riskantes Unterfangen dar« (ebd.: 7).

Diese postmoderne Identitäts-Bastelarbeit kann man als befreiend empfinden und mit entsprechend gewählter Musik zelebrieren. Wer sich dadurch aber grundlegend verunsichert fühlt, der sucht wohl zumindest im Bereich der Kultur verlässliche Identifikationsangebote:

»Individuals celebrate authenticity in order to balance the extreme dislocation that characterizes life in the postmodern world, in which traditional concepts of self, community and space have collapsed. This collapse has led to a widespread internalization of doubt and an obsession with distinguishing the real from the fake« (Lewin/Williams 2009: 66).

3. Seit der Erfindung von realitätsnahen audiovisuellen Aufzeichnungs- und Übertragungsmedien, insbesondere aber seit der flächendeckenden Verbreitung des Internets ist unser Weltverhältnis von zunehmender Medialisierung und Virtualisierung geprägt. Der Wunsch nach Liveness, handwerklicher und emotionaler Authentizität ist eine Reaktion auf diese Entwicklung. Er wächst mit der Seltenheit, mit der unmittelbare Erfahrungen heute überhaupt noch gemacht werden können. Bezeichnend ist, dass ausgerechnet Fernsehen und Internet als Symbole dieser Entwicklung uns gegenwärtig die größte Authentizität versprechen, das Live-dabei-Sein, das Besonders-nahdran-Sein, den voyeuristischen Blick hinter die Kulissen. Mit der Zunahme solcher Prozesse wird es nicht nur schwieriger, sondern annähernd unmöglich, noch »Echtes« zu finden. Wer heute zeigen will, dass er etwas Authentisches anzubieten hat, der muss es mit großem Aufwand medial inszenieren, damit er überhaupt wahrgenommen wird. 


\section{Literatur}

Amrein, Ursula (Hg.) (2009). Das Authentische. Referenzen und Repräsentationen. Zürich: Chronos.

Appen, Ralf von (2007). Der Wert der Musik. Zur Ästhetik des Populären (= texte zur populären Musik 4). Bielefeld: transcript.

Appen, Ralf von (2013). »Popmusik als Kunst.«In: Populäre Musik (= Kompendien Musik 14). Hg. v. Martin Pfleiderer, Ralf von Appen und Nils Grosch. Laaber: Laaber (im Druck).

Appen, Ralf von / Doehring, André (2006). »Nevermind The Beatles, here's Exile 61 \& Nico: -The top 100 records of all time - a canon of pop and rock albums from a sociological and an aesthetic perspective. «In: Popular Music 25, H. 1, S. 21-39.

Arnu, Titus (2012). »Heimspiel im Wohnzimmer.«In: Süddeutsche Zeitung vom 15. Mai, S. 9.

Auslander, Philip (1999). Liveness. Performance in a Mediatized Culture. London, New York: Routledge.

Barker, Hugh / Taylor, Yuval (2007). Faking it. The Quest for Authenticity in Popular Music. New York, London: Norton.

Billboard (2010). »Top 25 Tours.« In: Billboard.com, http://www.billboard. com/features/top-25-tours-of-2010-1004134022.story\#/ features/top-25-toursof-2010-1004134022.story (Zugriff am 19.8.2012).

Billboard (2011). »2011 - The Year in Music. 25 Top Tours.« In: Billboard.com, http://www.billboard.com/features/top-25-tours-of-2011-1005641362.story\#/ features/top-25-tours-of-2011-1005641362.story (Zugriff am 19.8.2012).

Bönisch, Dana (2009). »Pet Shop Boys. Jenseits von Jedem.«In: Intro, Nr. 170 (April), S. 8-9.

Chiapello, Ève (2010). »Evolution und Kooption. Die >Künstlerkritik < und der normative Wandel. «In: Kreation und Depression. Freiheit im gegenwärtigen Kapitalismus. Hg. v. Christoph Menke und Juliane Rebentisch. Berlin: Kadmos, S. 38-51.

Diaz-Bone, Rainer (2002). Kulturwelt, Diskurs und Lebensstil. Eine diskurs-theoretische Erweiterung der bourdieuschen Distinktionstheorie. Opladen: Leske + Budrich.

Dibben, Nicola (2009). „Vocal Performance and the Projection of Emotional Authenticity. «In: The Ashgate Research Companion to Popular Musicology. Hg. v. Derek B. Scott. Farnham: Ashgate, S. 317-333.

Eickelpasch, Rolf / Rademacher, Claudia (2004). Identität. Bielefeld: transcript.

Fischer-Lichte, Erika (2000). »Theatralität und Inszenierung. « In: Inszenierung von Authentizität. Hg. v. Isabel Pflug. Tübingen, Basel: Francke, S. 31-44.

Fischer-Lichte, Erika (2004). Ästhetik des Performativen. Frankfurt/M.: Suhrkamp.

Fischer-Lichte, Erika (2005a). Art. »Aufführung." Metzler Lexikon Theatertheorie. Hg. v. Erika Fischer-Lichte, Doris Kolesch und Matthias Warstat. Stuttgart, Weimar: Metzler, S. 16-26.

Fischer-Lichte, Erika (2005b). Art. »Inszenierung. «In: Metzler Lexikon Theatertheorie. Hg. v. Erika Fischer-Lichte, Doris Kolesch und Matthias Warstat. Stuttgart, Weimar: Metzler, S. 146-153.

Fornäs, Johan (1994). »Listen to your Voice! Reflexivity and Authenticity in Rock, Rap and Techno Music. In: New Formations 24, S. 155-173. 
Friedman, Ted (1993). »Milli Vanilli and the Scapegoating of the Inauthentic. In: Bad Subjects 9: The Music Issue, http://bad.eserver.org/issues/1993/09/ friedman.html (Zugriff am 11.6.2012).

Frith, Simon (1987). "Towards an Aesthetic of Popular Music." In: Music and Society. Hg. von Richard Leppert und Susan McClary. New York: Cambridge University Press, S. 133-149.

Frith, Simon / Horne, Howard (1987). Art into Pop. London, New York: Methuen.

Funk, Wolfgang / Krämer, Lucia (Hg.) (2011). Fiktionen von Wirklichkeit. Authentizität zwischen Materialität und Konstruktion. Bielefeld: transcript.

Funk, Wolfgang (2011). "Seltsame Schleifen und wahrhaftiges Erzählen - Authentizität im zeitgenössischen englischsprachigen Roman.« In: Fiktionen von Wirklichkeit. Authentizität zwischen Materialität und Konstruktion. Hg. v. dems. und Lucia Krämer. Bielefeld: transcript, S. 225-244.

Goodwin, Andrew (1988). "Sample and Hold. Pop Music in the Digital Age of Reproduction. «In: Critical Quarterly 30, Nr. 3, S. 34-49.

Grossberg, Lawrence (1992). We gotta get out of this place. Popular Conservatism and Postmodern Culture. New York u. London: Routledge [dt. Übersetzung mit dem Untertitel Rock, die Konservativen und die Postmoderne. Wien: Löcker 2010].

Jooß-Bernau, Christian (2010). Das Pop-Konzert als para-theatrale Form. Seine Varianten und seine Bedingungen im kulturell-öffentlichen Raum. Berlin: de Gruyter.

Jost, Christofer (2011). »U2 und die >Realität der Massenmedien<. Zur Transmedialität populärer Musik. «In: Populäre Musik, mediale Musik? Transdisziplinäre Beiträge zu den Medien der populären Musik. Hg. v. Christofer Jost, Daniel Klug, Axel Schmidt und Klaus Neumann-Braun. Baden-Baden: Nomos, S. 147-175.

Kolesch, Doris (2005). Art. "Liveness. «In: Metzler Lexikon Theatertheorie. Hg. v. Erika Fischer-Lichte, Doris Kolesch und Matthias Warstat. Stuttgart, Weimar: Metzler, S. 188-190.

Kotarba, Joseph. A. (2009). »Pop Music as a Resource for Assembling an Authentic Self: A Phenomenological-Existential Perspective. «In: Authenticity in Culture, Self, and Society. Hg. v. Phillip Vannini und J. Patrick Williams. Farnham: Ashgate, S. 153-168.

Lewin, Philip / Williams, J. Patrick (2009). »The Ideology and Practice of Authenticity in Punk Subculture. «In: Authenticity in Culture, Self, and Society. Hg. v. Phillip Vannini und J. Patrick Williams. Farnham: Ashgate, S. 65-83.

Lindholm, Charles (2008). Culture and Authenticity. Malden, MA: Blackwell.

McCarthy, E. Doyle (2009). »Emotional Performances as Dramas of Authenticity.« In: Authenticity in Culture, Self, and Society. Hg. v. Phillip Vannini und J. Patrick Williams. Farnham: Ashgate, S. 241-255.

Menke, Christoph (2005). »Innere Natur und soziale Normativität. Die Idee der Selbstverwirklichung.«In: Die kulturellen Werte Europas. Hg. v. Hans Joas und Klaus Wiegandt. Bonn: Bundeszentrale für politische Bildung, S. 304-352.

Menke, Christoph / Rebentisch, Juliane (2010). "Vorwort: Zum Stand ästhetischer Freiheit « In: Kreation und Depression. Freiheit im gegenwärtigen Kapitalismus. Hg. v. dens. Berlin: Kadmos, S. 7f.

Moore, Allan (2002). »Authenticity as Authentication. In: Popular Music 21, Nr. 2, S. 209-223.

N.N. (1995). »Jede Note klar und sauber.«In: Der Spiegel, H. 37, S. 200-206. 
Nelson, Paul (1965). »Newport Folk Festival, 1965«. In: Sing Out (November 1965), zit. n. Wiederabdruck in The Pop, Rock, and Soul Reader. Histories and Debates. Hg. v. David Brackett. Oxford, New York: Oxford University Press, S. 131-133.

Pareles, Jon (1990). »The Midi Menace: Machine Perfection is Far from Perfect. «In: The New York Times vom 13. Mai, http://www.nytimes.com/1990/05/13/arts/ pop-view-the-midi-menace-machine-perfection-is-far-form-perfect.html? pagewanted=all\&src=pm (Zugriff am 19.8.2012).

Pattie, David (2007). Rock Music in Performance. Houndmills, New York: Palgrave Macmillan.

Pattison, Robert (1987). The Triumph of Vulgarity. Rock Music in the Mirror of Romanticism. Oxford u. a.: Oxford University Press.

Reinartz, Joe (2010). »Top Tours 2010.« In: Pollstar.com, http://www.pollstar. com/news_article.aspx?ID=751701 (Zugriff am 19.8.2012).

Rössner, Michael / Uhl, Heidemarie (Hg.) (2012). Renaissance der Authentizität? Über die neue Sehnsucht nach dem Ursprünglichen. Bielefeld: transcript.

Seel, Martin (2007). »Inszenieren als Erscheinenlassen.« In: Ders., Die Macht des Erscheinens. Frankfurt/M.: Suhrkamp, S. 67-81.

Smith, Jay (2011). »2011 Top Tours - U2 Reigns Supreme.« In: Pollstar.com, http: / /www.pollstar.com/news_article.aspx?ID=751701 (Zugriff am 19.8.2012).

Stimpfle, Alois (2011). ") Ich habe den Herrn gesehen (Joh 20,18) - Zur Authentizität biblischer Wirklichkeitskonstruktion am Beispiel der Auferweckung Jesu. « In: Fiktionen von Wirklichkeit. Authentizität zwischen Materialität und Konstruktion. Hg. v. Wolfgang Funk und Lucia Krämer. Bielefeld: transcript, S. 157-178.

Taylor, Charles (2007). A Secular Age. Cambridge, MA: Harvard University Press.

Touré (2011). »Adele. Pop's Biggest Voice of 2011 Runs on Cigarettes, Red Wine and High-octane Heartbreak. «In: Rolling Stone, Nr. 1129 (28. April), S. 52-56.

Vannini, Phillip / Williams, J. Patrick (Hg.) (2009). Authenticity in Culture, Self, and Society. Farnham: Ashgate.

Weinstein, Deena (1999). »Art versus Commerce. Deconstructing a (Useful) Romantic Illusion. «In: Stars Don't Stand Still in the Sky. Music and Myth. Hg. v. Karen Kelly und Evelyn McDonnell. London: Routledge, S. 56-69.

\section{Filmographie}

Adele (2011). Live at the Royal Albert Hall [CD/DVD]. XL Recordings XLDVD559.

Black Eyed Peas (2011). "Super Bowl Half Time Show«, http://www.youtube. com/watch?v=zjz5H1ZrOuc.

Coldplay (2009). »Green Eyes« [Sydney 2009], http://www.youtube.com/watch?v=xRkwQ61s1A.

Foo Fighters (2011). Wasting Light Live From 606, http://www.youtube.com/ watch? $=$ Xnmzins2Uow.

Grohl, Dave (2012). »Dave Grohl Speech - Grammys 2012«, http://www.daily motion.com/video/xomnj5_dave-grohl-speech-grammys-2012-foo-fighters-win-b est-rock-performance-for-walk_lifestyle.

Kablitz-Post, Cordula (2009). Deutschland, deine Künstler: Campino [Dokumentarfilm], Erstausstrahlung: Das Erste am 16.7.2009, 22:45-23:30.

Metallica (1998). Cunning Stunts [DVD]. Universal, Vertigo 0602498702260.

Roxy Music (1972). »Ladytron« [BBC 2 Old Grey Whistle Test], http://www. dailymotion.com/video/x38qqp_roxy-music-ladytron-ogwt-1972_music. 
Roxy Music (1973). »Re-Make, Re-Model« [Radio Bremen Musikladen vom 30. Mai], http: / /www.youtube.com/watch?v=FlvCDotJBRs.

Perry, Katy (2011). »Hot N Cold at Rock in Rio 2011«, http://www.youtube. com/watch?v=Xc7PBBTDYaA.

Pet Shop Boys (2010). Pandemonium [CD/DVD]. Parlophone 5099962808323.

Die Toten Hosen (2008). »Wort zum Sonntag / Bonnie \& Clyde« [Rock am Ring], http: / /www.youtube.com/watch?v=49-7njhbbCk\&feature=related.

Die Toten Hosen (2009). »Paradies« (Berlin, 3. Juli), http://www.youtube.com/ watch? $v=a y 0 d h 60 t 3 M A$.

Die Toten Hosen (2012). »Das Magical-Mystery-Tour 2012 Videotagebuch«, http:// www.dietotenhosen.de/neuigkeiten-news-magical-mystery-tour-2012-videotage buch-teil-1-hamburg.php.

West, Kanye (2012). »Runaway« (London, 18. Mai), http://www.youtube.com/ watch?v=4pRAHLwsOYo\&feature=related.

ZDF Aspekte (2012). Sendung vom 20. April, 23:00 Uhr, http://www.zdf.de/ZDFme diathek/beitrag/video/1624382/aspekte-vom-20.-April-2012\#/beitrag/video/16 24382/aspekte-vom-20.-April-2012.

\begin{abstract}
This paper discusses the concepts of authenticity, liveness, and staging in reference to live performances of popular music. It aims to analyze social and individual functions of authenticity, and to develop awareness for the different strategies employed to suggest authenticity on stage.

In the first section, the author differentiates between four types of authenticities: personal, socio-cultural, executional, and emotional. He adopts definitions of liveness and staging from theater studies, applies them to the field of popular music, and shows how the ideals of liveness and authenticity are necessarily in conflict with the staged character of public performances because spectators can never be sure about the extent to which performances are enacted or reveal something »authentic. «

In a second section, current stage performances of such differing acts as Metallica, Die Toten Hosen, Pet Shop Boys, and Adele are analyzed to show how musicians deal with this conflict and how they try to fulfill expectations concerning all four dimensions of authenticity. Their strategies include, among others, performing »unplugged, « leaving room for moments of interaction and spontaneity, using downsized stages, or frankly exposing the artificial character of the performance (»authentic inauthenticity«).

Overall, the analyses show that authenticity - no matter how staged it is - is still a very important value in different styles of popular music. The paper closes with considerations about deeper cultural reasons for the increased longing for authenticity in the arts.
\end{abstract}

